Arch. hist. jap. Vol. 14, n. 1 (Februar 1958).

S. $125-132$.

Anat. Inst. d. Med. Fak., Univ. Okayama (Vorstand: Prof. M. SEKI).

\title{
Über die Kern-Plasmarelation der Leberzellen und Rückenmuskel- fasern des Huhnes in der Zeit der Entwicklung und über die der verschiedenen Skeletmuskelfasern der erwachsenen weißen Ratte nach der CHALKLEY-HAUGschen Treffermethode.
}

\begin{abstract}
Chalkley-Haug 氏当的百分率算定法による発育中の家鴊の肝細胞と背筋 線維及び成長した大黒鼠の諸㮔骨格筋線維の核-胞形質比について.
\end{abstract}

Hiroaki IWAKUMA 岩 隈 博 明.

(Eingegangen am 8. Dezember 1957.)

Es gibt über die quantitative analyse des Zellkerns schon eine ziemlich reichliche Literatur. Vor allem ist hier die Kern-Plasmarelation von R. HERTWIG (1903) zu nennen. Diese Relation soll uns zur Orientierung über die Funktion des Kerns dienen, um so mehr als neuerdings die quantitative Analyse von Nukleinsäure im Kern vielfach studiert wird. Die bisher gewöhnlich gebrauchte Methode zur Ermittelung der Relation ist folgende. Man zeichnet die mikroskopischen Bilder der Zellen und Kerne mit dem Zeichnenapparat auf ein Papier oder photographiert sie und mißt entweder den Flächeninhalt der Bilder mit dem Planimeter oder schneidet die Umrißzeichnung der Zellen und Kerne, wiegt diese und berechnet die ausgeschnittenen Anteile. Weil aber diese Methode ziemlich umständlich ist, erfreut sie sich keiner besonderen Beliebtheit.

CHALKLEY (1943) fand eine umwälzende, einfache Methode zur Ermittelung des relativen Flächeninhaltes bestimmter Gewebselemente im histologischen Schnitt. Diese , hit 'Methode wurde an den gleichmäßig gebauten parenchy matösen Geweben, der Leber des Kaninchens und der Nierenrinde und Hypophyse der Maus angewandt. In Deutschland nannte HAUG (1955) dieses Messungsverfahren Treffermethode. Er probierte sie optisch-physikalisch und konnte danach den Grauzellkoeffizient der Hirnrinde bestimmen. IVERSEN und THAMSEN (1956) bestimmten nach der gleichen Methode die Kern-Plasmarelation der Leberzellen der normalen Maus in verschiedenen Altern und stellten die Veränderlichkeit derselben im Laufe des Lebens fest. IVERSEN (1956) konnte ferner die Veränderung der Kern-Plasmarelation in der regenerierenden Rattenleber konstatieren.

Durch die Mitteilung von HAUG angeregt habe ich nach dieser Methode die Bestimmung der Kern-Plasmarelation der Leberzellen und Rückenmuskelfasern des Huhnes während der Entwicklung und die der verschiedenen Skeletmuskelfasern der erwachsenen weißen Ratte vorgenommen.

\section{Material.}

Es wurden als erstes Material 5, 6, 7, 8, 9, 10, 12, 15 und 20 Tage alte Hühnerembryonen und 3 und 70 Tage alte Hühnchen gewählt. Man fixierte die Objekte 
in 10\%igem Formalin etwa 2 Wochen lang und bettete sie in Paraffin ein, um Serienschnitte von $10 \mu$ Dicke zu verfertigen und diese mit Hämatoxylin und Eosin zu färben.

Als zweites Material brauchte man eine männliche weiße Ratte von $182 \mathrm{~g}$ Gewicht. Das Tier wurde mit Chloroform getötet und im Eisschrank bei $2-4^{\circ} \mathrm{C}$ 7 Stunden lang aufbewahrt. Man fixierte die Muskeln, ohne Trennung aus dem Skelett, in 10\%igem Formalin etwa 1 Woche lang. Erst nach dem Härten in 7094\% igem Alkohol wurden dem Tiere 16 Arten von Skeletmuskeln entnommen und in Celloidin eingebettet. Die Querschnitte von $15 \mu$ Dicke wurden mit Hämatoxylin und Eosin gefärbt.

\section{Methode.}

Die Schnitte wurden unter dem Mikroskop in 1500facher Vergrößerung mit der CHALKLEY-HAUGschen Treffermethode gemessen. Dabei wurde das Immersionsobjektiv von 100facher Vergrößerung und von der Apertur 1.25 mit der Kombination vom Okular von 15 facher Vergrößerung angewandt.

Das Prinzip der Methode ist kurz gesagt folgendes. Das Verhältnis der Flächeninhalte der verschiedenen Gewebs- bzw. Zellenanteile in dem Schnittpräparat wird in einer sehr dünnen Brennebene des Mikroskops durch die Frequenz ihres Treffens mit dem im Okular eingesetzten Trefferpunkt gesucht. Nach der originalen CHALKLEYschen Methode wurde als die Trefferpunkte die Endpunkte von vier langen und einem kurzen Haaren, die auf der Leiste im Okular angesetzten waren, benutzt. IVERSEN und THAMSEN (1956) benutzten ein Glasscheibchen, auf welchem man gleicherweise eingerichtete Linien eingeschnitten hatte. HAUG (1955) hat die Methode durch die Einführung einer Okularzählscheibe mit einem großen Quadrat, das durch gekreuzte Linien in 100 kleinere untergeteilt ist, präzisiert. Unter Benutzung von 121 durch Kreuzung der Linien entstandenen Punkten werden in 100 Sehfeldern 12100 Treffer bekommen. Ich arbeitete mit der HAUGschen Okularzählscheibe, und zwar benutzte ich beim Hühnchen 49 Kreuzpunkte der Linien, welche in der Mitte des Sehfeldes kleine $6 \times 6$ Quadrate begrenzen, und bei der weißen Ratte alle 121 Kreuzpunkte der Linien.

Die Dicke der Linien auf der Okularzählscheibe war etwa $0.4 \mu$ in 1500 facher Vergrößerung, so daß der Flächeninhalt jedes Kreuzpunktes etwa $0.16 \mu^{2}$ betrug. Die als Trefferpunkte benutzten Kreuzpunkte der Linien stellten sich in der Brennebene des Mikroskopes. Bei der Ausführung der Messung wurde nach Abschluß einer Beobachtung das Bild des Schnittes von der Brennebene ausgenommen und dann der Schnitt aufs Geratewohl umgezogen, um ein neues Bild wieder in die Brennfläche zu bringen. Dieses Verfahren wiederholte man immer wieder. Man registierte jedesmal die Frequenz der Treffungen auf den Kern, das Cytoplasma bzw. andere Gewebselemente. Wenn dabei der Treffer nicht deutlich auf die äußere Grenzf läche des Kerns traf, so rechnete man mit einem Treffen auf das Cytoplasma. Die Probabilität der Frequenz des Treffens auf einen Gewebsbestandteil wird selbständlich nur durch die Größe seines gesamten Flächeninhaltes bestimmt. Daher ist das Verhältnis der Frequenz des Treffens an den Kern zu der an das Cytoplasma die gesuchte Kern-Plsmarelation. 
Die Bestimmung des Treffenprozentes des Kerns und Cytoplasmas wurde so lange fortgesetzt, bis man einen konstanten Wert bekam. Diesen Zustand erreichte man in der Mehrzahl der Fälle nach 2,000 bis 3,000 maligem Treffen. Bei den Skeletmuskeln der weißen Ratte mußten aber, weil die Zahl des Treffens an den Kern viel kleiner war, 12,100 Treffen gezählt werden.

Bezüglich der Dicke der Schnitte hat CHALKLEY (1943) an den 1, 3, 5, 10 und $15 \mu$ dicken Schnitten gezeigt, daß der Fehlergrenze des ausgewerteten Wertes der Kern-Plasmarelation, verglichen mit dem Mittelwert, weniger als $3 \%$ beträgt. Der ermittelte Wert ist also praktisch von der Dicke der Schnitte nicht abhängig.

CHALKLEY (1943) benutzte eine hochgradige Vergrößerung bei der Mikroskopie. HAUG (1955) hat die zuläßige Vergrößerungsgrenze für die Treffermethode bestimmt, indem er die objektive Tiefenschärfe bei der Kombination des Objektivs und Okulars von verschiedenen Vergrößerungsvermögen sowie die subjektive Tiefenschärfe des Beobachters von verschiedenen Altern einrechnete. Danach sollen die kleinste Tiefenschärfe etwa $0.4 \mu$ und ihre höchste Grenze für die Treffermethode $1 \mu$ sein. Nach der von ihm angegebenen Formel $1, T=n_{0} \cdot\left(\frac{4 \lambda}{A^{2}} æ_{\alpha}+\frac{S}{A V} \omega\right)$, berechnet, beträgt die objektive Tiefenschärfe $0.52 \mu$ bei meiner 1500 fachen Vergrößerung in kombinierter Anwendung des Objektivs von numerischer Apertur 1.25 und 100 facher Vergrößerung und von dem Okular von 15 facher Vergrößerung $(T$ : die Tiefenschärfe oder der Tiefenbereich, $A$ : numerische Apertur des Objektives, $V$ : die Gesamtvergrößerung, $S$ : der Sehabstand $250000 \mu(25 \mathrm{~cm}), \lambda$ : die durchschnittliche Wellenlänge des Lichtes $0.55 \mu, æ_{a}$ : die Konstante von der Größe 1/8, $n_{0}$ : der Brechungsindex der meisten histologischen Gewebsteile 1.45). In der Praxis wird die nach dieser Formel berechnete Tiefenschärfe durch die Nachakkomodation vergrößert. Diese Vergrößerung läßt sich nach der HAUGscher Formel 2, $T^{\prime}=n_{0} \cdot \frac{S^{2}}{V^{2}} \cdot\left(\frac{1}{S_{1}}-\frac{1}{S_{2}}\right)$, berechnen $\left(T^{\prime}:\right.$ die Vergrößerung von $\mathrm{T}$ durch die Nachakkomodation, $S_{1}$ : die untere Akkomodationsgrenze, $S_{2}$ : die obere Akkomodationsgrenze, $\frac{1}{S_{1}}-\frac{1}{S_{2}}$ : die individuelle unterschiedliche Akkomodationsbreite des Auges. Bei der Untersuchung mit normalsichtigem Auge mit dem unendlich weiten Fernpunkt $S_{2}=\infty$ und $\frac{1}{S_{2}}=0$. Für die Berechnung wird also $S_{1}$ allein zuständig). Hierbei ist $S_{1}$ vom Alter abhängig und beträgt für mein Alter etwa $150,000(15 \mathrm{~cm})$. Deshalb ist $T^{\prime} 0.27 \mu$ auszurechnen. Daher wird bei meinem Fall die subjektive Tiefenschärfe $0.79 \mu$.

\section{Ergebnisse und Auswertung.}

Die nach der oben erwähnten Methode ermittelten Ergebnisse über die KernPlasmarelation verschiedener Zellen lassen sich im folgenden zusammenfassen.

\section{A. Die Leberzellen des Huhnes während der Entwicklung.}

Die gefundenen Werte sind in Tabelle 1 zusammengestellt. Man ersieht daraus, daß die Kern-Plasmarelation am 5.-6. Tage der Bebrütung am größten ist, indem sie 29.1 und 31.9 beträgt, dann mit fortschreitender Brütung bis zum 3. Lebenstage 
Tabelle 1. Kern-Plasmarelation der Leberzellen des Huhnes während der Entwicklung.

\begin{tabular}{|c|c|c|c|c|}
\hline \multirow{2}{*}{\multicolumn{2}{|c|}{ Alter }} & \multirow{2}{*}{$\begin{array}{l}\text { Nach d. } \\
\text { Treffer- } \\
\text { methode }\end{array}$} & \multicolumn{2}{|c|}{ Nach d. Zeichnenapparatmethode } \\
\hline & & & $\mathrm{M} \pm \mathrm{m}$ & $\mathrm{V}$ \\
\hline \multirow{9}{*}{ Brüttage } & 5 & 29.1 & $33.6 \pm 1.2$ & 24.4 \\
\hline & 6 & 31.9 & $32.4 \pm 0.9$ & 23.7 \\
\hline & 7 & 27.3 & $28.3 \pm 0.8$ & 21.9 \\
\hline & 8 & 26.5 & $26.0 \pm 0.9$ & 23.8 \\
\hline & 9 & 23.7 & $22.9 \pm 0.6$ & 20.1 \\
\hline & 10 & 21.2 & $23.4 \pm 0.7$ & 20.5 \\
\hline & 12 & 21.0 & $22.2 \pm 0.9$ & 28.3 \\
\hline & 15 & 17.7 & $22.0 \pm 0.9$ & 28.6 \\
\hline & 20 & 17.0 & $19.7 \pm 0.6$ & 22.3 \\
\hline \multirow{2}{*}{$\begin{array}{l}\text { Lebens- } \\
\text { tage }\end{array}$} & 3 & 15.5 & $16.4 \pm 0.7$ & 30.5 \\
\hline & 70 & 19.4 & $20.0 \pm 0.7$ & 25.0 \\
\hline
\end{tabular}

ständig kleiner wird und zum Schluß 15.5 beträgt. Am 70. postnatalen Tage wird die Relation wieder etwas größer, nämlich 19.4. Die Relation scheint aber zeitweise je nach dem Funktionszustand der Leber zu schwanken. IVERSEN u. a. (1956) haben ähnliche Werte bezüglich der Kern-Plasmarelation der Leberzellen an der normalen Maus nach der Treffermethode gewonnen. Sie konstatierten, daß die Relation nach der Geburt bis zum 90. Tage schnell abnimmt und etwa am 500. Tage wieder ein wenig größer wird.

Um diese nach der Treffermethode gefundenen Werte mit denen nach anderer Methode gefundenen zu vergleichen, wurde die folgende Untersuchung ausgeführt. Man zeichnete das mikroskopische Bild mit dem ABBEschen Zeichnenapparat auf ein Papier und maß die Flächeninhalte des Kerns und Cytoplasmas der Zellen mit Hilfe des Planimeters, und zwar die von 50 Zellen in jeder Altersstufe. Die somit gewonnenen Werte sind ebenfalls in Tabelle 1 angegeben. Die Relationswerte nach

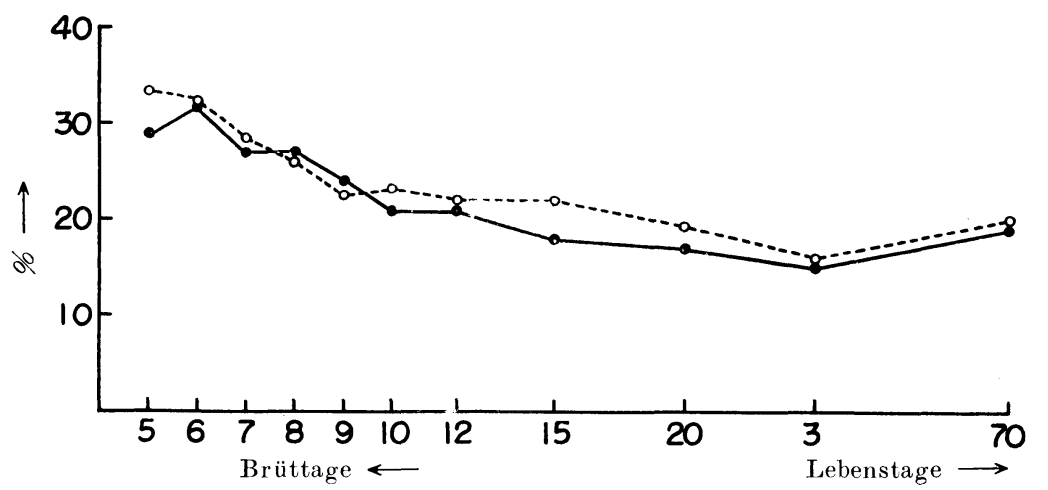

Abb. 1. Kern-Plasmarelation der Leberzellen des Huhnes während der Entwicklung. - - nach der Treffermethode, $\circ \ldots \ldots \ldots . . . .0$ nach der Zeichnenapparatmethode. 
den beiden Methoden sind auch vergleichend in Abb. 1 dargestellt. Man sieht, daß die Fehlergrenze der nach der Trffermethode gewonnenen Werte im Bereiche der Standardfehler (m) der mit dem Zeichnenapparat erhaltenen liegen. Die Resultate nach der Treffermethode sind also recht befriedigend, zumal wenn man in Rechnung zieht, daß die Zahl der gemessenen Zellen klein war. Zu bemerken ist die Tatsache, daß die nach der Treffermethode gefundenen Werte etwas kleiner sind als die nach dem Zeichnungsverfahren gewonnenen. Das beruht offenbar darauf, daß bei der Zeichnung nur gewisse Zellen, beim Treffungsverfahren aber alle Zellen ohne Auswahl in Betracht kamen. Daß Gewebselemente ohne Auswahl zur Untersuchung kommen, ist ein großer Vorteil der Treffermethode, neben dem anderen, daß diese Methode sehr einfach und daher für kompliziert strukturierte Gewebe auch anwendbar ist. Der einzige, aber nicht kleine Nachteil dieser Methode ist, daß die nach dieser Methode gewonnenen Werte keine absolute Zahlen, sondern relative sind, worauf auch HAUG hingewiesen hat.

\section{B. Die Fasern der Rückenmuskeln des Huhnes während der Entwicklung.}

Man ermittelte die Relation des Kerns zum Plasma der Rückenmuskelfasern des Huhnes in der Entwicklung in den Querschnitten des Muskels. Die Ergebnisse sind in Tabelle 2 und Abb. 2 gezeigt. Die Kern-Plasmarelation ist am Anfang der Brütung vom größten Wert 37.1-44.1 und reduziert sich während der Entwicklung ständig, bis sie am 70. Lebenstage nur 3.0 beträgt. Dieses Resultat steht in weitgehender Übereinstimmung mit dem der vorherigen Messung vom Verfasser nach einer anderen Methode (1957), daß nämlich der Durchmesser der Rückenmuskelfasern sich mit dem Zeitablauf allmählich vermehrt und nach der Geburt beträchtlich größer wird, während der Kern sich am Ende der Embryonalzeit und nach der Ausbrütung verkleinert.

\section{Die Fasern von verschiedenen Skeletmuskeln der erwachsenen weißen Ratte.}

Nach der Untersuchung von HIRAI (1942) sind die Skeletmuskeln beim sich lebhafter bewegenden Wildkaninchen meistens dünner als beim trägen Hauskaninchen, und bei Kaninchen führen diejenigen Muskeln, die Daur- oder Haltefunktionen ausüben, in der Regel dünnere Fasern. HIRAI (1943) hat später sehr viele Skeletmuskeln der weißen Ratte untersucht und auch hier die letztere Tatsache bestätigt. 


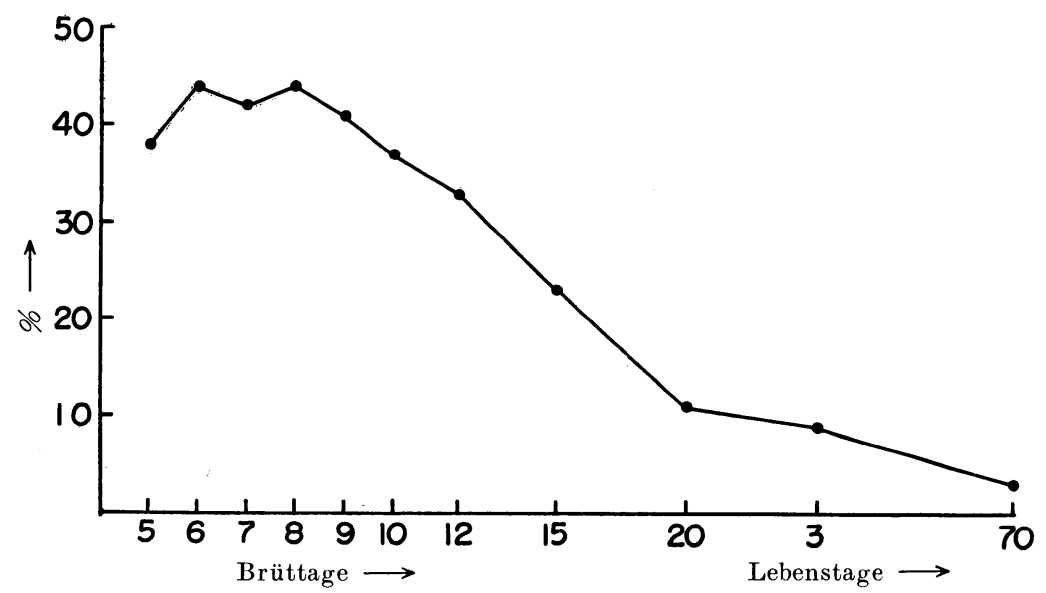

Abb. 2. Kern-Plasmarelation der Rückenmuskelfasern des Huhnes während der Entwicklung nach der Treffermethode.

Danach sind gewöhnlich die Fasern der Kopf- und Halsmuskeln feiner als die der Bauch- und Hinterextremitätenmuskeln.

Die Kern-Plasmarelation der Fasern von 16 Arten Skeletmuskeln der weißen Ratte wurde nun nach der Treffermethode bestimmt. In Tabelle 3 sind die gefun-

Tabelle 3. Kern-Plasmarelation der Fasern von verschiedenen Skeletmuskeln der erwachsenen weißen Ratte.

\begin{tabular}{|c|c|c|c|c|c|}
\hline \multirow[b]{2}{*}{ Name der Muskern } & \multicolumn{3}{|c|}{ Getroffene Zahl } & \multirow{2}{*}{$\begin{array}{l}\text { K-P-Relation } \\
\left(\frac{\mathrm{K}}{\mathrm{P}} \times 100\right)\end{array}$} & \multirow{2}{*}{$\begin{array}{c}\text { Querschnitt } \\
\text { d. M.-fasern } \\
\text { d. Ratte v. } \\
210 \mathrm{~g} \\
\text { nach HIRAI } \\
\left(\mu^{2} / 100\right)\end{array}$} \\
\hline & $\begin{array}{c}\text { an } \\
\text { Cyto- } \\
\text { plasma }\end{array}$ & $\begin{array}{c}\text { an } \\
\text { Kern }\end{array}$ & $\begin{array}{c}\text { an } \\
\text { anderen } \\
\text { Teilen }\end{array}$ & & \\
\hline Rectus oculi temporalis & 11069 & 309 & 722 & 2.79 & 1.6 \\
\hline Diaphragma, Pars costalis & 11335 & 272 & 493 & 2.40 & 4.7 \\
\hline Rectus abdominis, Brustteil & 11432 & 233 & 435 & 2.04 & 7.9 \\
\hline Rhomboides major & 11634 & 214 & 252 & 1.84 & 6.5 \\
\hline Temporalis & 11567 & 191 & 342 & 1.65 & 4.3 \\
\hline Intercostalis internus $\mathrm{V}$ & 11819 & 193 & 88 & 1.63 & 11.0 \\
\hline Flexor carpi ulnaris & 11806 & 184 & 110 & 1.56 & 6.4 \\
\hline Masseter, Oberflächl. Teil & 11699 & 181 & 220 & 1.55 & 6.1 \\
\hline Sternomastoideus & 11741 & 159 & 200 & 1.35 & 7.9 \\
\hline Intercostalis externus $\mathrm{V}$ & 11583 & 152 & 365 & 1.31 & 9.0 \\
\hline Brachialis & 11332 & 147 & 621 & 1.30 & 8.6 \\
\hline Pectoralis major & 11707 & 145 & 248 & 1.24 & 8.9 \\
\hline Semimembranaceus & 11586 & 130 & 384 & 1.12 & 12.2 \\
\hline Quadratus lumorum & 11825 & 130 & 145 & 1.10 & 16.4 \\
\hline Tensor fasciae latae & 11657 & 122 & 321 & 1.05 & 15.6 \\
\hline Gastrocnemius, Caput fibulare & 11629 & 120 & 351 & 1.03 & 14.9 \\
\hline
\end{tabular}


denen Werte in der Rэihenfolge der Größe der Faserdicke der Muskeln zusammenstellt. In der gleichen Tabelle sind die von HIRAI (1943) gemessenen Flächeninhalte der Fasern der gleichnamigen Muskeln von seiner A-Ratte von $210 \mathrm{~g}$ Körpergewicht zum Vergleich angegeben. Die Kern-Plasmarelation ist bei den feinsten Muskelfasern von M. rectus oculi temporalis am größten und beträgt 2.79, demgegenüber ist die der dickeren Fasern von M. semimembranaceus, M. quadratus lumborum, M. tensor fasciae latae und M. gastrocnemius von dem kleinsten Wert 1.121.03 .

Das Diaphragma, das der Muskel mit anhaltender Tätigkeit ist, besteht aus dünnen Fasern, deren Kern-Plasmarelation groß und 2.40 ist. Die Kern-Plasmarelation von M. masseter, M. temporalis und Mm. intercostales ist kleiner als die der Lenden- und Hinterextremitätenmuskeln.

Im allgemeinen ist der Wert der Kern-Plasmarelation der feineren Muskelfasern mit mehr anhaltender Tätigkeit des Erwachsenen größer als die der dickeren, welche von Zeit zu Zeit schwere Arbeit leisten.

\section{Zusammenfassung.}

Die CHALKLEYsche Methode, die neulich von HAUG in Deutschland eingeführt und einer Prüfung unterzogen worden ist, wurde für unsere Untersuchung angewandt und ihre Zuverläßlichkeit festgestellt.

Nach dieser Methode wurde festgestellt, daß der Wert der Kern-Plasmarelation der Leberzellen des Huhnes während der Entwicklung allmählich abnimmt und am 3. postnatalen Tage am kleinsten wird, sich aber am 70. postnatalen Tage wieder ein wenig vergrößert. Die Kern-Plasmarelation der Rückenmuskelfasern ist am Anfang der Embryonalzeit groß, reduziert sich aber später rasch und wird am 70 . postnatalen Tage auffallend kleiner.

Der Wert der Kern-Plasmarelation der Skeletmuskelfasern der erwachsenen weißen Ratte ist in der Regel bei den immer arbeitenden Muskeln größer als bei den zeitweise kräftig arbeitenden Muskeln in der Lende und den hinteren Extremitäten. Bei den ersteren sind die Fasern meist dünner als bei den letzteren.

\section{内 容 自 抄.}

最近 Haug そより検討され,ドイツ国に紹介された Chalkley 氏法が再び検討さ れ, その信頼性が確められた。

この法によって調べると, 発育中の家鷄の肝細胞の核-胞形質比は年令と共に次 第に減少し, 生後 3 日で最も小さくなり, 生後70日で再び僅かに大きくなる. 又 その背筇線維の核一胞形質比は孵生の初期飞大で, 睬生の後期飞急激に減少し, 生 後70日では著しく小さくなる。

大黒鼠の骨格筋線維の核一胞形質比は常飞働く筋に大で, 時々大きな力を出す 腰部と後肢の筋飞小であり, 前者の筇線維は通例後者のものより細いから, 比較 的の核の大さは筋線維が細いほど大であるとも云える。 


\section{Literatur.}

Chalkley, H. W.: Method for the quantitative morphologic analysis of tissue. J. Nat. Canc. Inst. 4 (1943). - Haug, H.: Die Treffermethode, ein Verfahren zur quantitativen Analyse im histologischen Schnitt. Z. Anat. 118 (1955). - Hertwig, R.: Über Korrelation von Zell- und Kerngröße und ihre Bedeutung für geschlechtliche Differenzierung und die Teilung der Zelle. Biol. Zbl. 23 (1903). - Hirai, T.: Vergleichende histologische Untersuchungen der Skeletmuskeln des Haus- und Wildkaninchens. Okajimas Fol. anat. jap. 22 (1942). - Planimetrische Untersuchung der Skeletmuskelfasern des weißen Ratte. Okajimas Fol. anat. jap. 22 (1943). - Iversen, S.: The nucleo-cytoplasmic ratio in regenerating rat liver cells. Acta anat 27 (1956). - Iversen, S. a. A. Thamsen : The nucleo-cytoplasmic ratio in mouse liver cells. Acta path. microbiol. scand. 38 (1956). Iwakuma, H. : Messung der Zellen von verschiedenen Organen des Huhnes während der prä- und postnatalen Entwicklung. Arch. hist. jap. 12 (1957). 